\title{
The Relationship between Explanation and Patient Compliance in Hirudotherapy
}

\author{
Kwang Seog Kim ${ }^{1}$, \\ Ho Seup Sim, \\ Jun Ho Shin', \\ Jae Ha Hwang ${ }^{1}$, \\ Sam Yong Lee ${ }^{1}$
}

Departments of ${ }^{1}$ Plastic and Reconstructive Surgery and ${ }^{2}$ Preventive Medicine, Chonnam National University Medical School, Gwangju, Korea
Background: The use of leeches can effectively increase the salvage rate of flap congestion. However, the first reaction from patients and carers in using leeches in clinical fields is strong aversion. This can be due to the fact that development of our culture from agriculture to industrial society, coming across leeches became fairly rare. Also because of the biological traits that leeches carry; staying attached to a leg or other body parts of the host, sucking blood, and leaving wounds.

Methods: This study was conducted through questionnaires, divided into many subgroups. We scaled the compliance of the two therapies, with or without leech. Maximum scale of 10 showing no rejective response to the therapy and minimum scale of 0 showing the greatest rejective response.

Results: Overall subjects' compliance was improved after explaining the benefits of hirudotherapy. Irrelevant to the explanation, there was no significant difference in general compliance between male and female. Young-aged group and medical personnel or people studying medicine showed higher compliance over older-aged group and the general public.

Conclusion: In the terms of general social cognition, recognizing leech as a therapeutic material may not be welcomed at first, but provided with proper information and explanations, overall compliance of patients and carers can be improved and consequently result in superior outcomes in flap salvage.

Keywords: Surgical flaps / Bloodletting / Leeches / Patient compliance / Surveys and questionnaires
No potential conflict of interest relevant to this article was reported. microsurgical replantation and flap reconstruction, most patients and carers show aversion the first time they hear about this treatment.

The aim of this study is to understand people's general aversion to hirudotherapy, to analyze differences in the extent of aversion among subgroups, to examine how the extent of aversion changes after subjects receive a thorough explanation of hirudotherapy, and to explore methods of improving patient compliance.

\section{METHODS}

\section{Subjects and methods}

\author{
Department of Plastic and Reconstructive Surgery, Chonnam National University Medical \\ School, 42 Jebong-ro, Dong-gu, Gwangju 61469, Korea \\ E-mail: pskim@chonnam.ac.kr
}

Received August 24, 2017 / Revised September 19, 2017 / Accepted September 19, 2017
Hirudotherapy is a treatment using leeches that was approved by the FDA in 2004. Hirudotherapy utilizes the biological advantagon the blood of their host and during the course, release bloodthinning substances like anticoagulants along with their saliva [2] Following replantation or flap procedure, leeches are used to reAlthough there are many reports of leech therapy in the area of
A questionnaire was designed for a total of 200 subjects. In order 
to perform a subgroup analysis, we administered the questionnaire to 50 doctors, 50 medical students, and 100 non-medical personnel. In order to investigate the independent effect of explanation on the outcomes, we excluded subjects with prior knowledge that leeches could be used to improve postoperative outcomes in flap surgery and finger replantation; thus, the ten subjects who selected answer (1) for Question 6 were excluded, and the questionnaire was administered to another ten persons, to make two-hundred subjects in total.

\section{Questionnaire}

The questionnaire was constructed as follows. On Page 1, the aims of the study were not revealed to subjects to avoid bias in their responses. This page contained questions about subjects' information and 'pre-explanation compliance'. It consisted of the following items: (Fig. 1). 1. Sex, 2. Age, 3. Profession (doctor, medical student, or non-medical personnel), 4. Compliance to hirudotherapy on a scale of $0-10$.

Once subjects had completed their responses to Page 1, they were instructed to read the explanation and case reports on Pages 2 and 3. There were no time restrictions and, if required, subjects were provided with thorough explanations of difficult terms such as 'necrosis' and 'venous congestion'. The explanation was as follows.

\section{Explanation of the medical use of leeches (Fig. 2)}

'The skin is our body's primary defense, and it helps to maintain our health by preventing infections from entering the body. Defects in the skin and soft tissues can be caused by tumor removal, bedsores, or trauma, and these defects can be treated by disinfection, a negative pressure wound therapy system, skin graft, or flap surgery. Of these, flap surgery is a method of reconstructing severe tissue defects, and includes local flap surgery, regional flap surgery, and free flap surgery. For the flap to survive, it needs to be provided with an arterial blood supply and venous blood drainage. Even if the flap has a blood supply, once it has been oxygenated, it cannot survive if failure of the blood to drain causes venous congestion. Although leeches have been used in medicine since the distant past, people feel a sense of disgust at their appearance and their blood-feeding behavior. However, leaches are very useful for treating venous congestion, which is difficult to correct with surgery'

Next, the subjects were provided with images and an explanation about patients who had undergone breast reconstruction at our hospital using a transverse rectus abdominis myocutaneous flap, had been at risk of flap necrosis due to postoperative venous congestion, and had achieved a positive outcome by using hirudotherapy.

The first case introduced in the questionnaire. A 55-year-old woman who underwent breast-conserving surgery for left breast cancer 18 months previously had developed a soft tissue defect after 1 postoperative year due to infection of the inserted mesh (above). Reconstruction surgery was performed using a transverse rectus abdominis myocutaneous flap, but congestion in the medial margin of the flap led to a risk of necrosis. This was treated by hirudotherapy, and the flap color improved within 5 days (below, right).

The second case introduced in the questionnaire (Fig. 3). A woman underwent modified radical mastectomy for right breast cancer 8 years previously; reconstruction surgery was performed using a transverse rectus abdominis myocutaneous flap, but congestion developed in the upper, lateral, and inferior portions of the flap (above). Hirudotherapy was performed, and most of the congestion was alleviated within 6 days (below).

At the bottom of Page 3, the same questions as Page 1 were presented again, in order to check the subjects' 'post-explanation compliance'. Finally, a 6th question was presented to exclude subjects who had prior knowledge about hirudotherapy.

The improvement in compliance following the explanation was analyzed for all subjects. Subsequently, the subjects were divided into subgroups according to profession, sex, and age, and the improvement in compliance was analyzed for each subgroup.

In order to analyze the effects of profession, sex, and age on compliance, a one-way analysis of variance (ANOVA) was performed for the three conditions of pre-explanation, post-explanation, and change following explanation. Tukey's method was used for post-hoc testing. 


\section{Questionnaire on the relationship between explanation about hirudotherapy and patient compliance}

Hello, this is the Department of Plastic Surgery at Cheonnam University Hospital.

Using this questionnaire, our department is trying to investigate the relationship between explanation about the medical use of leeches (hirudotherapy) and compliance. We want to hear your opinions before and after reading the explanation and case studies below. The explanation can be found on the reverse side, but for the accuracy of our study, although it may be a little confusing, please complete the questionnaire on the front page before you read the explanation on the back.

If it is not too much inconvenience, we would be very grateful if you could sDare a little time to respond to our questionnaire.

Thank you.
1. What is your sex?
(1) Male (2) Female
2. How old are you?
(1) $20-50$ years (2) $30-40$ years (3) $40-60$ years (4) $50-60$ years

S. Are you a doctor or a medical student?

(1) Doctor (2) Medical student (3) Non-medical personnel

4. If you heard that you or a family member were to be treated with leeches, how would you rate your level of aversion?

(Compliance with no aversion would be given the highest possible score of 10; inability to comply due to severe aversion or diszust would be given the lowest possible score of 0 ) (1) (1) (2) (3) (4) (5) (6) (1) (8) (2)

(Once you have completed the questionnaire up to Question 4, Dlease read the explanation and case studies below)

Fig. 1. Questionnaire (1). 
(Bxplanation of the medical use of leeches)

The skin is our body's primery defense, and it helps to maintein our health by preventing infections from entering the body. Defects in the skin and soft tissues can be caused by tumor removal, bedsores. or traume, and these defects cen be treated by disinfection, a nezative pressure wound therapy system. skin ereft, or flap surzery. Of these, flap surzery is a method of reconstructing severe tissue defects. and includes locel flap surzery, rezional flap surzery, and free flap surzery. Por the flap to survive, it needs to be provided with an arterial blood supply and venous blood draineze. Bven if the flep has a blood supply. once it has been oxyreneted, it cennot survive if feilure of the blood to drein causes venous conzestion. Althourh leeches heve been used in medicine since the distent pest, people feel a sense of discust et their appeerence and their blood-feeding behavior. However, leeches are very useful for treatine venous conzestion, which is difficult to correct with surcery. With the development of arricultural societies into urben societies, it hes become less common to encounter leeches, and their appearence and blood-feeding behavior causes people to think of them as hermful animels. However, as expleined above, leeches can be used to effectively improve venous conzestion. We have experienced countless cases of treetment at our hospital, and without even discussing the results of academic studies. the patients receiving treatment and the doctors administering treatment heve witnessed its effectiveness with their own eyes.

(Case 1)

The imezes below are of en ectuel flep treeted at our hospitel using leeches.

In the preoperative imeze on the left, necrosis cen be observed sterting from the aree of dark skin on the left side of the photozraph. Looking closer, approximately one quarter of the whole flap near the aipple marzin is at risk of aecrosis, besed on its red discoloration, and the whole flep is at risk of venous conzestion.

The imaze on the right shows the flap after 8 deys of treatment using leeches. The aree at risk is now no more then one tenth of the whole flap. and the aree around the aipple, in particuler, no loazer appears at risk

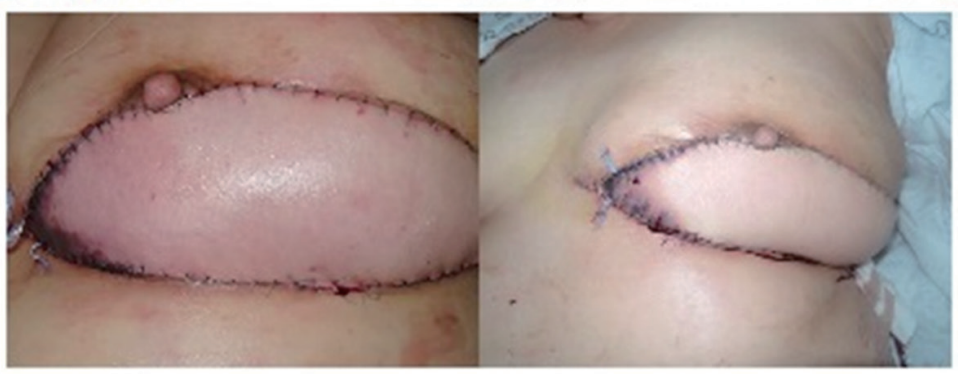

Fig. 2. Questionnaire (2). 


\section{(Case 2)}

This is a different case. The two images in the upper row show the flap immediately after surgery, while the image in the lower row shows the flap after using leeches. The black and dark red areas have been reduced considerably, and almost no necrosis can be observed after treatment; the flap has overall become more stable, negating the need for revision surgery.
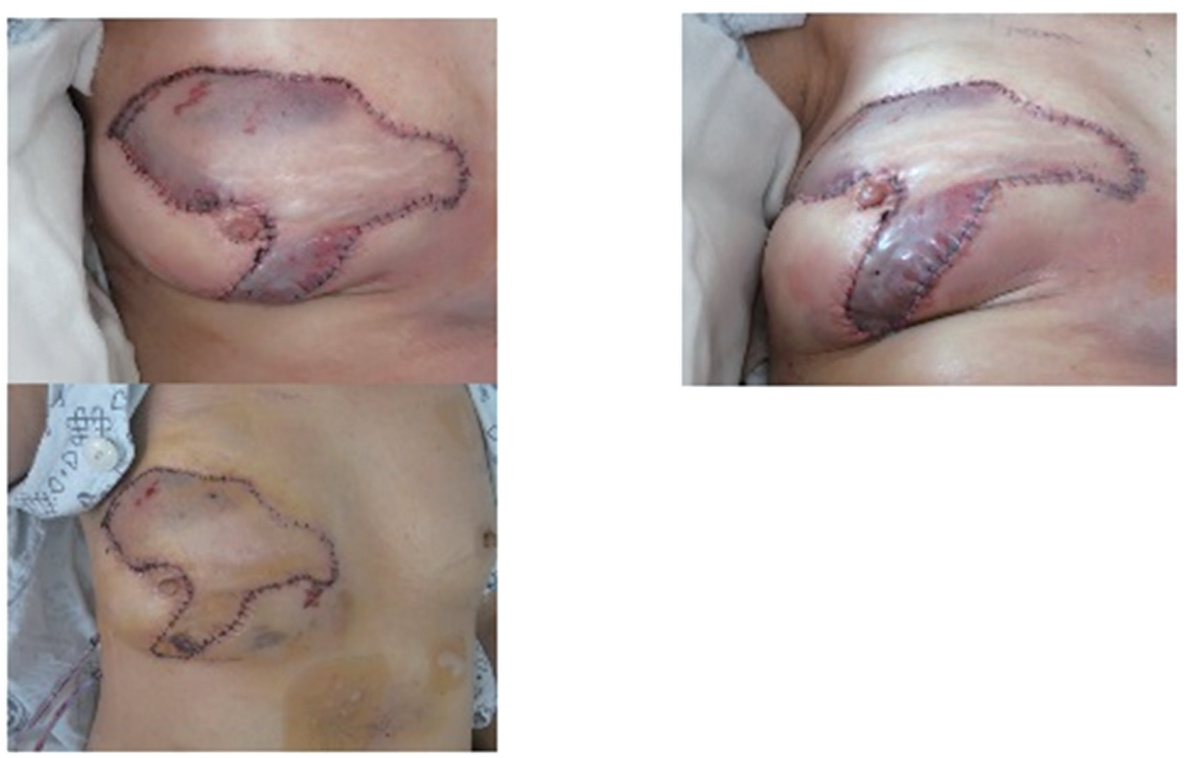

How is your compliance after looking at the two cases above and seeing the explanation about leech therapy?

5. If you heard that you or a family member were to be treated with leeches, how would you rate your level of aversion? (This is the same as Question 4)

(Compliance with no aversion would be given the highest possible score of 10; inability to comply due to severe aversion or diszust would be given the lowest possible score of 0 )

(1) (1) (2) (3) (4) (5) (6) (1) (8) (2) (1)

6. Did you already know that 'leeches could be used to improve postoperative outcomes in flap, reconstruction, or replantation surgery' before responding to this questionnaire?

(1) Yes (2) No

Thank you for responding to our questionnaire.

Fig. 3. Questionnaire (3). 
In order to investigate the effects of medical knowledge on compliance, the subjects were divided by profession, into subgroups of 50 doctors, 50 medical students, and 100 non-medical personnel.

In order to investigate the effects of sex on compliance, the 100 non-medical personnel were divided into subgroups of 48 males and 52 females. In order to investigate the effects of age on compliance, the 100 non-medical personnel were divided into subgroups of 29 subjects in their twenties, 20 subjects in their thirties, 27 subjects in their forties, and 24 subjects in their fifties.

\section{RESULT}

\section{Improvements in compliance by subgroup}

Among all subjects, compliance improved significantly after the explanation of hirudotherapy (Table 1).

Statistical analysis of the three subgroups by profession showed a significant improvement after the explanation of hirudotherapy (Table 2).

There was a significant improvement in compliance after the explanation of hirudotherapy irrespective of sex (Table 3 ).

There was also a significant improvement in compliance after the explanation of hirudotherapy in all age groups (Table 4).

\section{Comparison of improvement in compliance between subgroups}

In further statistical analysis of the three subgroups by profession, greater medical knowledge was associated with higher compliance before and after the explanation about hirudotherapy. Anal-

Table 1. Compliance before/after explanation

\begin{tabular}{lccccc} 
Compliance & No. & Mean & $\begin{array}{c}\text { Standard } \\
\text { deviation }\end{array}$ & $\begin{array}{c}\text { Correlation } \\
\text { coefficient }\end{array}$ & $p$-value ${ }^{\mathrm{a})}$ \\
$\begin{array}{c}\text { Compliance } \\
\text { before } \\
\text { explanation }\end{array}$ & 200 & 5.10 & 2.182 & 0.862 & $<0.001$ \\
$\begin{array}{c}\text { Compliance } \\
\text { after } \\
\text { explanation }\end{array}$ & 7.19 & 2.479 & & \\
\hline
\end{tabular}

a) Statistical significance was evaluated by T-test.
Table 2. Compliance before/after explanation compared with doctors, medical students and non-medical personel

\begin{tabular}{lcccc} 
Profession & No. & $\begin{array}{c}\text { Compliance } \\
\text { before } \\
\text { explanation } \\
\text { (SD) }\end{array}$ & $\begin{array}{c}\text { Compliance } \\
\text { after } \\
\text { explanation } \\
\text { (SD) }\end{array}$ & $p$-value \\
\hline Doctors & 50 & $6.64(2.058)$ & $9.14(1.591)$ & $<0.001$ \\
$\begin{array}{l}\text { Medical } \\
\text { students }\end{array}$ & 50 & $5.46(1.775)$ & $7.72(2.061)$ & $<0.001$ \\
$\begin{array}{l}\text { Non- } \\
\text { medicine }\end{array}$ & 100 & $4.14(1.928)$ & $5.95(2.320)$ & $<0.001$ \\
\hline
\end{tabular}

SD, standard deviation; Non-medicine, non-medical personel.

a)Statistical significance was evaluated by paired T-test.

Table 3. Lay public's compliance before/after explanation compared with male and female

\begin{tabular}{lcccc} 
Sex & No. & $\begin{array}{c}\text { Compliance } \\
\text { before } \\
\text { explanation } \\
\text { (SD) }\end{array}$ & $\begin{array}{c}\text { Compliance } \\
\text { after } \\
\text { explanation } \\
\text { (SD) }\end{array}$ & $p$-value ${ }^{\mathrm{a})}$ \\
\hline Male & 48 & $4.54(2.153)$ & $6.63(2.446)$ & $<0.001$ \\
\hline Female & 52 & $3.77(1.628)$ & $5.33(2.027)$ & $<0.001$ \\
\hline
\end{tabular}

SD, standard deviation.

a) Statistical significance was evaluated by paired T-test.

Table 4. Lay public's compliance before/after explanation compared with age groups

\begin{tabular}{ccccc}
\hline $\begin{array}{c}\text { Age } \\
\text { group }\end{array}$ & No. & $\begin{array}{c}\text { Compliance } \\
\text { before } \\
\text { explanation (SD) }\end{array}$ & $\begin{array}{c}\text { Compliance } \\
\text { after explanation } \\
\text { (SD) }\end{array}$ & $p$-value ${ }^{a}$ \\
\hline 20's & 29 & $5.48(1.595)$ & $7.24(2.081)$ & $<0.001$ \\
\hline 30's & 20 & $4.80(1.908)$ & $6.80(1.881)$ & $<0.001$ \\
\hline 40's & 27 & $3.56(1.502)$ & $5.41(2.171)$ & $<0.001$ \\
\hline 50 's & 24 & $2.63(1.377)$ & $4.29(1.922)$ & $<0.001$ \\
\hline
\end{tabular}

SD, standard deviation.

a)Statistical significance was evaluated by paired T-test.

ysis of the improvement in compliance showed no significant differences between doctors and medical students or between medical students and non-medical personnel, but there was a significant difference between doctors and non-medical personnel.

Statistical analysis of sex showed, a significant difference between men and women in pre-explanation compliance and postexplanation compliance. The change in compliance was also significantly different between men and women.

We also analyzed the pre- and post-explanation compliance, as well as the improvement in compliance, by age. Pre-explanation compliance showed no significant differences between subjects in 
the 20 s and subjects in their 30 s, or between subjects in their $40 \mathrm{~s}$ and subjects in their 50s. However, when subjects in their 20s and 30 s were merged into one group and subjects in their 40 s and $50 \mathrm{~s}$ were merged into one group, there was a significant difference between these two groups

\section{DISCUSSION}

The group of all subjects showed an improvement in compliance following the explanation, and there were also differences in the improvement according to medical knowledge, sex, and age.

First, in the analysis by medical knowledge, greater medical knowledge was associated with a greater improvement in compliance. Medical knowledge could impact a subject's understanding of the effects of leeches on the flap.

Second, in the analysis by sex, there was a significant difference in pre-explanation compliance between males and females. There were also significant differences between males and females in post-explanation compliance and in the change in compliance. Males showed significantly higher scores for pre-explanation compliance and post-explanation compliance, and also showed a significantly greater increase in compliance. This is thought to be due to differences in the extent of disgust felt towards leeches, and the leeches being received more positively by males than females.

Third, the analysis by age showed that pre-explanation compliance was higher for younger age groups. Post-explanation compliance showed a decrease in the average compliance with increasing age. Although there were no significant differences between subjects in their 20s and 30s, 30s and 40s, or 40s and 50s, there were significant differences between subjects in their 20s and 40s, and between subjects in their 30s and 50s. However, when the change in compliance was analyzed, there were no large differences between subjects in their $20 \mathrm{~s}$ (1.76 points), in their 30 s (2.00 points), in their $40 \mathrm{~s}$ (1.85 points), or in their $50 \mathrm{~s}$ (1.67 points), and the oneway ANOVA and post-hoc Tukey's tests also showed no significant difference in the change in compliance between the four groups. Age had no effect on the improvement in compliance. Subjects of younger age could be considered to be more flexible in taking on new scientific facts. However, sex ratios were not consistent among different age groups, which was a limitation during the sample design process. Since sex is a factor that can affect compliance, the results could be made more reliable if the sex ratios were the same across all age groups.

The use of leeches for flap venous congestion management shows different compliance according to the age and medical knowledge of patients and carers. Age, sex, and medical knowledge all showed an effect on the improvement in compliance. Of these, medical knowledge is the only factor that can be changed. Therefore, in order to increase compliance, if the medical knowledge of patients and carers were to be enhanced through continual, in-depth education, it should be possible to reduce their aversion to hirudotherapy.

\section{REFERENCES}

1. Pannucci CJ, Nelson JA, Chung CU, Fischer JP, Kanchwala SK, Kovach SJ, et al. Medicinal leeches for surgically uncorrectable venous congestion after free flap breast reconstruction. Microsurgery 2014; 34:522-6.

2. Porshinsky BS, Saha S, Grossman MD, Beery Ii PR, Stawicki SP. Clinical uses of the medicinal leech: a practical review. J Postgrad Med 2011;57:65-71.

3. Praveen N, Deepa CK, Laxminarayan B. Hirudotherapy for flap salvage-report of two cases. Int J Adv Case Rep 2015;2:471-3. 